\title{
Turbulence and intermittent transport at the boundary of magnetized plasmas
}

Garcia, O.E.; Naulin, V.; Nielsen, A.H.; Juul Rasmussen, J.

Published in:

Physics of Plasmas

Link to article, DOI:

$10.1063 / 1.1925617$

Publication date:

2005

Document Version

Publisher's PDF, also known as Version of record

Link back to DTU Orbit

Citation (APA):

Garcia, O. E., Naulin, V., Nielsen, A. H., \& Juul Rasmussen, J. (2005). Turbulence and intermittent transport at the boundary of magnetized plasmas. Physics of Plasmas, 12(6), 062309. https://doi.org/10.1063/1.1925617

\section{General rights}

Copyright and moral rights for the publications made accessible in the public portal are retained by the authors and/or other copyright owners and it is a condition of accessing publications that users recognise and abide by the legal requirements associated with these rights.

- Users may download and print one copy of any publication from the public portal for the purpose of private study or research.

- You may not further distribute the material or use it for any profit-making activity or commercial gain

- You may freely distribute the URL identifying the publication in the public portal

If you believe that this document breaches copyright please contact us providing details, and we will remove access to the work immediately and investigate your claim 


\title{
Turbulence and intermittent transport at the boundary of magnetized plasmas
}

\author{
O. E. Garcia, ${ }^{*}$ V. Naulin, A. H. Nielsen, and J. Juul Rasmussen \\ Association EURATOM-Risф National Laboratory, \\ OPL-128 Ris $\phi$, DK-4000 Roskilde, Denmark
}

(Dated: March 31, 2005)

\begin{abstract}
Numerical fluid simulations of interchange turbulence for geometry and parameters relevant to the boundary region of magnetically confined plasmas are shown to result in intermittent transport qualitatively similar to recent experimental measurements. The two-dimensional simulation domain features a forcing region with spatially localized sources of particles and heat outside which losses due to motion along open magnetic field lines dominate, corresponding to the edge region and the scrape-off layer, respectively. Turbulent states reveal intermittent eruptions of hot plasma from the edge region, propagating radially far into the scrape-off layer in the form of field-aligned filaments, or blobs. This results in positively skewed and flattened single-point probability distribution functions of particle density and temperature, reflecting the frequent appearance of large fluctuations. The conditional fluctuation wave-forms and transport statistics are also in good agreement with those derived from experiments. Associated with the turbulence bursts are relaxation oscillations in the particle and heat confinement as well as in the kinetic energy of the sheared poloidal flows. The formation of blob structures is thus related to profile variations, which are here triggered in a quasi-periodic manner by a global dynamical regulation due to self-sustained sheared flows.
\end{abstract}




\section{INTRODUCTION}

The success of particle and heat confinement in magnetized plasma experiments crucially depends on the control of transport in the outermost plasma region in contact with material surfaces. This regulates the particle and heat loads on the plasma-facing components, which must be controlled for a stable operation of the next generation high-power confinement devices. An understanding of the physics governing transport at the boundary of magnetized plasmas is thus crucial but at present far from complete. ${ }^{1}$

Recently, there have been a large number of experimental investigations demonstrating the appearance and motion of strongly non-linear plasma objects resulting in intermittent convective transport in the edge and scrape-off layer (SOL) regions of magnetic confinement devices. ${ }^{2-28}$ These structures, commonly referred to as "blobs", are observed as magnetic field-aligned filaments of excess particles and heat as compared to the ambient plasma, propagating radially outwards from the plasma column. The radial propagation makes these structures different from the large-scale vortices and conditional structures observed in several basic plasma experiments. ${ }^{29-34}$ The blob structures have amplitudes significantly exceeding that of the ambient plasma and large radial velocity components. This causes bursty transport events and anomalous single-point probability distribution functions. It is of interest to note that such filaments have been explicitly observed by advanced imaging techniques, ${ }^{25-28}$ and appear in a wide range of experimentally accessible confinement states. In fact, also the fine structure of edge localized modes seem to share the defining characteristics of blobs. ${ }^{35-39}$ Another intriguing result is that similar fluctuation characteristics are found for a variety of magnetic geometries, including linear devices, ${ }^{12-14}$ reversed field pinches, ${ }^{9-11}$ stellarators, ${ }^{4-8}$ and tokamaks. ${ }^{13-24}$ Intermittent SOL transport due to coherent plasma structures thus seems to be a universal phenomenon independent of the details of the magnetic geometry as well as the underlying instability mechanisms by which these structures are generated. This important observation has been a strong motivation for the basic approach taken in the study presented here.

A pronounced feature of all the above mentioned experiments is the presence of positively skewed and flattened probability distribution functions (PDFs) of single-point particle density and temperature recordings in the SOL, indicating the high probability for large fluctuations above the background level. ${ }^{11-23}$ Moreover, as revealed by conditional averaging, there is a clear temporal asymmetry of these signals with a predominance of sharply rising and slowly decaying 
waveforms. ${ }^{14-22}$ For tokamak boundary plasmas the structures radial size and propagation velocity are estimated to be up to a few centimeters and one tenth of the acoustic velocity close to the last closed flux surface (LCFS). These values, as well as the fluctuation amplitudes, decay as the structures propagate through the SOL, consistent with particle losses along open magnetic field lines. ${ }^{16-20}$

Theoretical work related to blob transport in SOL plasmas has mostly been based on simple analytical models describing the transient radial propagation of isolated plasma blobs. ${ }^{40-46}$ For toroidally magnetized plasmas this was suggested to be due to particle drifts caused by the nonuniform magnetic field, resulting in a vertical charge polarization and hence radial advection by the electric drift. ${ }^{40}$ The non-linear evolution and radial propagation of isolated blob structures was recently demonstrated by numerical simulations in Ref. 42 , also confirming the asymmetric conditional wave-forms experimentally derived from single probe recordings. On the other hand, selfconsistent modeling and numerical simulations also describing the formation of such structures has remained elusive. Previous two-dimensional simulations have relied on the thin layer approximation and assumed a local turbulence drive within the SOL region, ${ }^{47-53}$ while three-dimensional simulations have usually assumed frozen profiles and are not amenable for long integration times in order to make advanced statistical analysis. ${ }^{54,55}$

In the present paper we report on two-dimensional numerical simulations demonstrating the formation and non-linear evolution of blob structures and their influence on plasma transport in the SOL of toroidal plasmas. Our model is based on the fluid description and governs the selfconsistent evolution of the particle density, temperature and vorticity at the outboard mid-plane of a toroidally magnetized plasma. The driving instability is the interchange mode due to an inhomogeneous magnetic field. In order to facilitate long run simulations and statistical data analysis, we ignore parallel drift wave dynamics as well as magnetic shear and consider only electrostatic perturbations, pertinent to the low pressure plasma at the boundary. On the other hand, we retain the fully non-linear structure of the flow compression terms which is mandatory in order for the model to conserve particles and energy. This further allows us to account for profile evolution and to describe the order unity perturbations to the background plasma parameters that is observed in the experiments.

The model equations are discussed in Sec. II, with particular attention to topics such as the drift approximation, diamagnetic cancellation, quasi-neutrality, particle and energy conservation, and the thin layer approximation. Different from most of the previous work, we model the spatially 
separated source and sink regions of particles and heat through what corresponds to the LCFS. The plasma fueling and heating is localized inside this radial position, while on the outside a linear damping of all dependent variables is invoked to model particle losses along open magnetic field lines. The time-averaged particle density and temperature profiles are thus peaked in the edge region inside the LCFS and are relatively flat throughout the SOL. The resulting pressure gradient drives interchange motions in the edge region, leading to radial propagation of localized structures identified as blobs. Moreover, the associated convective cells generate sheared poloidal flows, resulting in a state of bursting in the global fluctuation level and relaxation oscillations in the particle and heat confinement as well as the mean poloidal flow energy integral. ${ }^{56-60}$ Associated with the large fluctuation levels are eruptions of hot plasma from the edge region inside the LCFS, propagating far into the SOL. As discussed in Sec. III, careful statistical analysis of the turbulence simulation data yields results in excellent agreement with that obtained from experimental measurements. In particular, we observe single-point probability distribution functions with large skewness and flatness factors in the SOL, asymmetric conditional wave-forms for the particle density and the radial velocity with a steep front and a trailing wake, and a maximum conditional radial blob velocity of one fifth of the acoustic speed. The two-dimensional contour plots reveal the fine spatial details of the blobs, showing a tail left behind the front of the mushroom-like structures typical for interchange turbulence and in agreement with animations from fast camera gas puff imaging. ${ }^{28}$ Also the transport statistics are in close correspondence with experimental measurements. A discussion of our results and conclusions are given in Sec. IV. A first report of this study was presented in Ref. 60 .

\section{MODEL EQUATIONS}

In this section we present a derivation and discussion of the model equations applied in the present work. We base our description on the interchange instability of a non-uniformly magnetized plasma. In the case of an unstable equilibrium, the drive of the fluctuating motions is a convective transport of particles and heat outwards along the major radius of the toroidally magnetized plasma. ${ }^{61,62}$ A self-consistent description of this collective dynamics is here considered as a mechanism for intermittent turbulence in the plasma boundary region. In order to perform long-run simulations producing sufficient data to allow detailed statistical analysis, we reduce our description to two spatial dimensions and include parallel transport along open magnetic field lines 
as simple linear damping terms. We begin our discussion by considering the collective cross-field dynamics and neglect dissipation and parallel motions until further notice. Readers not interested in these analytical elaborations may readily jump to Sec. II F for an overview of the model equations used in this work.

\section{A. Drift approximation}

Reduced fluid model equations for low-frequency dynamics in magnetized plasmas are all based on the drift approximation whereby the momentum equation for each charged particle species is reduced to an algebraic expression for the fluid drifts in terms of scalar fields. The first step is to derive an implicit expression for the cross-field fluid drift for any species of particle charge $q$ and mass $m$,

$$
\mathbf{v}_{\perp}=\frac{1}{B} \mathbf{b} \times \nabla \phi+\frac{1}{q n B} \mathbf{b} \times \nabla p+\frac{m}{q B} \mathbf{b} \times\left(\frac{\partial}{\partial t}+\mathbf{v} \cdot \nabla\right) \mathbf{v}
$$

where $\mathbf{b}=\mathbf{B} / B$ denotes the unit vector along the magnetic field and we have assumed a scalar pressure field $p$ and consider only electrostatic perturbations through the electric potential $\phi$. Augmented with the particle continuity equation for each species,

$$
\frac{\partial n}{\partial t}+\nabla \cdot(n \mathbf{v})=0
$$

and the temperature equation by introducing a closed form of the cross-field heat flux $\mathbf{q}_{\perp}$,

$$
\frac{3}{2} n\left(\frac{\partial}{\partial t}+\mathbf{v} \cdot \nabla\right) T+n T \nabla \cdot \mathbf{v}+\nabla \cdot \mathbf{q}_{\perp}=0,
$$

a closed model in terms of scalar fields is then obtained by an iteration procedure, neglecting some of the terms on the right hand side of equation (1) to lowest order. The first term on the right hand side is the familiar electric drift $\mathbf{v}_{E}$ while the second term is the diamagnetic drift $\mathbf{v}_{d}$, defined respectively by

$$
\mathbf{v}_{E}=\frac{1}{B} \mathbf{b} \times \nabla \phi, \quad \mathbf{v}_{d}=\frac{1}{q n B} \mathbf{b} \times \nabla p .
$$

The latter comprises diamagnetic effects due to particle gyration as well as fluid motions corresponding to guiding center drifts due to a non-uniform magnetic field. ${ }^{62}$ Indeed, inhomogeneous and curved magnetic fields make the lowest order drifts compressible. In particular, for the electric drift we have

$$
\nabla \cdot \mathbf{v}_{E}=\nabla\left(\frac{1}{B}\right) \cdot \mathbf{b} \times \nabla \phi+\frac{1}{B} \nabla \times \mathbf{b} \cdot \nabla \phi=C(\phi)
$$


where we in the last equality have introduced the curvature operator $C$. For low pressure plasmas the two terms in the first equality are equal. Introducing an ordering and the corresponding lowest order drift, a closed form of the cross-field fluid motion is obtained by iteration of Eq. (1). The last term on the right hand side is then generally referred to as the polarization drift.

\section{B. Diamagnetic cancellation}

As mentioned above, only the compressible part of the diamagnetic drift describes collective motions. In a uniform magnetic field the diamagnetic fluxes are incompressible, reflecting diamagnetism due to particle gyration. Hence the diamagnetic drifts do not contribute to any advection process, a general property applying to all moment equations and loosely referred to as diamagnetic cancellation. Consider first the compression of the lowest order particle fluxes,

$$
\nabla \cdot\left(n \mathbf{v}_{E}+n \mathbf{v}_{d}\right)=\mathbf{v}_{E} \cdot \nabla n+n C(\phi)+\frac{1}{q} c(p)
$$

revealing that only the electric drift contributes to the fluid advection. A similar diamagnetic cancellation occurs in the heat equation. Using the collective cross-field heat flux $\mathbf{q}_{\perp}=5 n T \mathbf{b} \times$ $\nabla T / 2 q B$ and the exact relations

$$
\begin{aligned}
\frac{3}{2} n \mathbf{v}_{d} \cdot \nabla T & =\frac{3 T}{2 q B} \mathbf{b} \cdot \nabla n \times \nabla T, \\
n T \nabla \cdot \mathbf{v}_{d} & =\frac{T}{q B} \mathbf{b} \cdot \nabla n \times \nabla T+\frac{T}{q} \mathcal{C}(p), \\
\nabla \cdot \mathbf{q}_{\perp} & =-\frac{5 T}{2 q B} \mathbf{b} \cdot \nabla n \times \nabla T+\frac{5 n T}{2 q} C(T),
\end{aligned}
$$

we readily find that the diamagnetic drift does not contribute to heat advection, leaving only compressional terms due to a non-uniform magnetic field in Eq. (3),

$$
\frac{3}{2} n \mathbf{v}_{d} \cdot \nabla T+n T \nabla \cdot \mathbf{v}_{d}+\nabla \cdot \mathbf{q}_{\perp}=\frac{7 n T}{2 q} \mathcal{C}(T)+\frac{T^{2}}{q} \mathcal{C}(n) .
$$

A similar but more lengthy calculation, involving the gyro-viscous stress tensor, shows that the diamagnetic drift does not contribute to the advective non-linearity in the polarization drift as well. As will be discussed in the following, a closed model is then obtained by assuming quasi-neutrality and forming a generalized vorticity equation by subtracting the particle continuity equations for ions and electrons. 


\section{Quasi-neutrality}

Based on the electric drift to lowest order, the polarization current density obtained by summing over all particle species becomes

$$
\mathbf{J}_{p}=-\frac{\rho_{m}}{B} \mathbf{b} \times\left(\frac{\partial}{\partial t}+\mathbf{v}_{E} \cdot \nabla\right) \mathbf{v}_{E}
$$

where $\rho_{m}$ is the plasma mass density. If we for the sake of demonstration assume a homogeneous plasma and a uniform magnetic field, the electric charge compression due to this current is given by

$$
\nabla \cdot \mathbf{J}_{p}=-\frac{\rho_{m}}{B^{2}}\left(\frac{\partial}{\partial t}+\mathbf{v}_{E} \cdot \nabla\right) \nabla_{\perp}^{2} \phi
$$

This should be compared to the expression describing advection of space charges by the electric drift,

$$
\frac{\partial \rho}{\partial t}+\nabla \cdot\left(\rho \mathbf{v}_{E}\right)=\varepsilon_{0}\left(\frac{\partial}{\partial t}+\mathbf{v}_{E} \cdot \nabla\right) \nabla^{2} \phi
$$

where $\rho$ is the space charge density. The advection of space charges thus contributes with terms similar to but a factor $\varepsilon_{0} B^{2} / \rho_{m}$ less than the compression due to the polarization drift. This factor is usually a large number for magnetized plasmas. Thus the assumption of quasi-neutrality allows to neglect the direct role of space charges, while the electric fields are governed by the quasi-neutrality condition $\nabla \cdot \mathbf{J}=0$. What remains is to perform a consistent iteration in the drift approximation to provide a well-defined reduced fluid model. Here we will assume cold ions and neglect electron inertia to achieve a model that is analytically and computationally tractable.

\section{Particle and energy conservation}

The collective dynamics described by the reduced model should conserve the number of particles as well as the fluid energy. From the particle continuity equation (2) and the temperature equation (3) we derive a conservation equation for the thermal energy density,

$$
\frac{\partial}{\partial t}\left(\frac{3}{2} p\right)+\nabla \cdot\left(\frac{3}{2} p \mathbf{v}_{E}+\frac{1}{B} \mathbf{b} \times \nabla \frac{5 p T}{2 q}\right)+p \mathcal{C}(\phi)=0 .
$$

Integrating this pressure equation over space, assuming the boundary integrals to vanish, gives the evolution of the heat confinement,

$$
\frac{d}{d t} \int d \mathbf{x} \frac{3}{2} p=-\int d \mathbf{x} p C(\phi) .
$$


The term on the right hand side describes a collective energy transfer due to a non-uniform magnetic field, which notably alters the confinement of thermal energy. Let us consider the sign of this transfer term for a magnetic field $B(R)=B_{0} R_{0} / R$ directed opposite to the toroidal axis whose coordinate is denoted by $\Theta$ and $B_{0}$ is the field strength on the magnetic axis at major radius $R_{0}$. In terms of the elementary cylindrical coordinates $(R, \Theta, Z)$, the energy transfer term in Eq. (13) is simply given by

$$
\int d \mathbf{R} p \mathcal{C}(\phi)=-\int d \mathbf{R} \frac{2 p}{B R} \frac{\partial \phi}{\partial Z}
$$

which is nothing but a geometric weight of the collective thermal energy flux along the major radius, with $Z$ the coordinate along the major torus axis and $d \mathbf{R}=R d R d \Theta d Z$ is the differential volume element. The sign of the transfer term is thus such as to reduce the particle and heat confinement provided the non-linear heat flux is on average radially outwards with respect to the torus axis.

The interchange motion that decreases the plasma pressure results in an increase of the kinetic energy due to the electric drift of the plasma. Indeed, there is a conservative transfer from heat to fluctuation kinetic energy due to the non-uniform magnetic field. To demonstrate this we multiply the quasi-neutrality condition $\nabla \cdot \mathbf{J}=0$ with the electrostatic potential. Introducing the cross-field electric current density $\mathbf{J}=\mathbf{J}_{p}+\mathbf{J}_{d}$ where $\mathbf{J}_{d}=\mathbf{b} \times \nabla p / B$ is the diamagnetic current, this may be written as

$$
-\mathbf{J}_{p} \cdot \nabla \phi+\nabla \cdot(\phi \mathbf{J})-\mathbf{J}_{d} \cdot \nabla \phi=0
$$

For a well-defined fluid closure the first term will integrate to yield the time rate of change of the kinetic energy corresponding to the electric drift in addition to a transport term. ${ }^{63,64}$ The second term also has the form of a transport term, while the last term on the left hand side provides nothing but the transfer rate present in the heat equation (12) in addition to yet another transport term,

$$
\mathbf{J}_{d} \cdot \nabla \phi=-\mathbf{v}_{E} \cdot \nabla p=-\nabla \cdot\left(p \mathbf{v}_{E}\right)+p \mathcal{C}(\phi)
$$

In summary, collective transport along the major radius of a toroidally magnetized plasma directly affects the confined thermal energy by an conservative energy transfer to kinetic energy of the electric drifts. This important feature of interchange motions is usually violated in reduced descriptions of collective plasma motions in non-uniform magnetic fields. 


\section{E. Thin layer approximation}

An approximation commonly invoked in the derivation of reduced fluid models is that of a thin plasma layer such that the relative change in particle density and temperature is small in the domain under consideration. The main motivation for this simplification is that it allows to neglect variations in the particle density in the compression of the lowest order drifts, and thus significantly reduces the necessary computational resources for numerical solutions of the vorticity or charge continuity equation. To reveal the implications of this assumption, let us consider a thin plasma layer of depth $d$ at the minor radial position $r_{0}$, such that the radial domain is restricted with $r-r_{0}$ in the range between 0 and $d$ where $d / r_{0} \ll 1$, known as the thin layer or local approximation. Introducing local slab coordinates by $x=r-r_{0}$ and $y=r_{0} \theta$ with $\theta$ the poloidal angle, the full particle density may locally be written as

$$
n=\mathcal{N}+\triangle n \frac{d-x}{d}+\eta
$$

where $\mathcal{N}$ is a uniform background density, $\triangle n$ is the density variation over the plasma layer in the basic state and $\eta$ describes the fluctuations as well as the turbulence modifications of the equilibrium density profile. Clearly, for weak variations around the background, $\eta / \mathcal{N} \sim \triangle n / \mathcal{N} \ll 1$, the two last terms on the right hand side of Eq. (17) may be neglected to lowest order. Moreover, defining the density profile scale length by $1 / L_{n}=-\left(\partial n_{0} / \partial x\right) / \mathcal{N}$ with $n_{0}(x)$ the equilibrium density, the particle density in Eq. (17) may be rewritten as

$$
\frac{n}{\mathcal{N}}=1+\frac{d-x}{L_{n}}+\frac{\eta}{\mathcal{N}}
$$

showing that the assumption of weak density variations implies considering a plasma layer whose thickness $d$ is much less than the equilibrium plasma density scale length. An important consequence of this approximation is that the compression terms $n \nabla \cdot \mathbf{v}$ appearing in the continuity equations may be linearized in the fluctuating velocities. Moreover, we may neglect the density variations in the polarization current, an approximation that is invoked in nearly all reduced fluid models and will be made here as well. However, contrary to previous work, we will retain the full non-linear structure of the compression terms due to the lowest order drifts in order to allow order unity perturbations to the background plasma parameters. 


\section{F. Reduced model equations}

In the following we will consider a local slab equilibrium for an inhomogeneous magnetic field $\mathbf{B}=B(x) \widehat{\mathbf{z}}$. We will neglect the density and magnetic field variation in the polarization drift, leading to the conventional form of the vorticity equation. At this point we introduce the so-called Bohm normalization defined by

$$
\omega_{c i} t \rightarrow t, \quad \frac{\mathbf{x}}{\rho_{s}} \rightarrow \mathbf{x}, \quad \frac{e \phi}{\mathcal{T}} \rightarrow \phi, \quad \frac{n}{\mathcal{N}} \rightarrow n, \quad \frac{T}{\mathcal{T}} \rightarrow T,
$$

where $c_{s}=\left(\mathcal{T} / m_{i}\right)^{1 / 2}$ is the acoustic velocity, $\omega_{c i}=e \mathcal{B} / m_{i}$ the ion gyration frequency at a characteristic magnetic field strength $\mathcal{B}, \rho_{s}=c_{s} \omega_{c i}$ the hybrid thermal gyration radius, and $\mathcal{N}$ and $\mathcal{T}$ are characteristic values of the particle density and electron temperature, respectively. Taking $\mathcal{B}$ as the normalization of the magnetic field, the exact inverse toroidal field $\mathcal{B} / B=1+r \cos \theta / R_{0}$ is approximated by $1+\varepsilon+\zeta x$ in our non-dimensional units. Here $\varepsilon=r_{0} / R_{0}$ is the inverse aspect ratio, $\zeta=\rho_{s} / R_{0}$, and $R_{0}$ is the major radius of the toroidal plasma. Defining the vorticity $\Omega=\nabla_{\perp}^{2} \phi$ and assuming cold ions we finally get the reduced model equations used in this study,

$$
\begin{gathered}
\frac{d n}{d t}+n \mathcal{C}(\phi)-c(n T)=\Lambda_{n}, \\
\frac{d T}{d t}+\frac{2 T}{3} \mathcal{C}(\phi)-\frac{7 T}{3} \mathcal{C}(T)-\frac{2 T^{2}}{3 n} \mathcal{C}(n)=\Lambda_{T}, \\
\frac{d \Omega}{d t}-\mathcal{C}(n T)=\Lambda_{\Omega},
\end{gathered}
$$

Here we have introduced the advective derivative with the electric drift and the curvature operator, defined respectively by

$$
\frac{d}{d t}=\frac{\partial}{\partial t}+\frac{1}{B} \widehat{\mathbf{z}} \times \nabla \phi \cdot \nabla, \quad c=-\zeta \frac{\partial}{\partial y} .
$$

On the right hand side of all equations we have added terms representing sources, sinks and collisional diffusion. Note that in the vorticity equation (20c) the first and second terms on the left hand side correspond to the divergence of the polarization and diamagnetic currents, respectively.

The influence of sinks, sources and dissipation is described by the terms on the right hand side of the above equations. For the particle density this is given by

$$
\Lambda_{n}=S_{n}(x)+D_{n} \nabla_{\perp}^{2} n-\sigma_{n}(x)(n-1),
$$

where $S_{n}$ is a radially localized particle source, $D_{n}$ is the collisional diffusion coefficient, and $\sigma_{n}$ represents linear damping due to particle transport along open magnetic field lines in the SOL. 
A similar form of the source and sink terms also applies to the temperature field. In order to avoid numerical problems due to very steep profiles, the thermodynamic fields are in the SOL damped to a constant background level which is by definition unity in the non-dimensional model. On the other hand, the vorticity is damped to zero while there is no vorticity source, that is, $\Lambda_{\Omega}=D_{\Omega} \nabla_{\perp}^{2} \Omega-\sigma_{\Omega} \Omega$.

We envisage that the blob structures are elongated along the ambient magnetic field but with a finite parallel correlation length due to the ballooning effect imposed by the toroidal geometry. With an average distance $2 \pi q R_{0} / 3$ to the end plates and a parallel velocity given by half the acoustic speed, the amplitude of the sheath damping coefficients within the present normalization is simply $3 \zeta / 4 \pi q$ where $q$ is the safety factor. The sheath damping coefficients are taken to be the same for all variables, except for the temperature field which is damped five times stronger due to the predominant loss of hot electrons through the end sheaths in the region of open magnetic field lines. We take the profiles of the source $S_{n}$ and sheath damping $\sigma_{n}$ to be

$$
\begin{aligned}
S_{n}(x) & =\left(\frac{2}{\pi}\right)^{1 / 2} \frac{I_{n}}{\delta_{s}} \exp \left(-\frac{x^{2}}{2 \delta_{s}^{2}}\right), \\
\sigma_{n}(x) & =\frac{\lambda_{n}}{2}\left[1+\tanh \left(\frac{x-x_{l}}{\delta_{l}}\right)\right],
\end{aligned}
$$

and similarly for the temperature. In the simulations presented here we further take the radial line integral $I$ of the particle and heat sources to be the same, with the sources located at the left hand side of the simulation domain, $\delta_{s}=\sqrt{2}$. The size of the simulation domain is given by $L_{x}=2 L_{y}=200$, and the sheath damping operates from the LCFS at $x_{l}=50$ with a sharp transition, $\delta_{l}=1$. A sketch of the simulation domain is presented in Fig. 1. In order to control the collective and collisional fluxes of particles and heat through the radial boundaries we apply the boundary conditions

$$
\phi=0, \quad \Omega=0, \quad \frac{\partial n}{\partial x}=0, \quad \frac{\partial T}{\partial x}=0,
$$

at $x=0$, while for the outer boundary at $x=L_{x}$ we use $n=T=1$, thus defining the characteristic particle density and temperature for this system. Periodic boundary conditions are invoked for the poloidal direction. The model thus governs the non-linear evolution of the interchange instability in the presence of strong radial asymmetry due to the spatially localized sources $S$ and sheath damping coefficients $\sigma$.

Our modeling of parallel losses is different from the conventional "sheath dissipation" terms used in many previous two-dimensional models, including the recent blob theories. ${ }^{40,41}$ There are 
at least three limitations to that standard model. First, the classical two-dimensional sheath dissipation model assumes perfectly connected field-aligned structures terminated by the end sheaths at material walls. This is in contradiction to the strong ballooning character of the fluctuations as observed in experiments. ${ }^{27}$ Second, the field-line averaging leading to a two-dimensional model cannot be rigorously justified for the non-linear advection and compression terms. Finally, it is not possible within this model to self-consistently describe the transition between open and closed field lines. We also note that the standard sheath dissipation model preferentially damps large spatial length scales, a property that has severe consequences for any predictions. A more careful treatment of the parallel transport along open field lines requires a three-dimensional model.

\section{G. Confinement and mean profiles}

The profile of any field, in the following denoted by a zero sub-script, is defined as its spatial average over the poloidal direction. Thus, the particle density and poloidal flow profiles are defined by

$$
n_{0}=\frac{1}{L_{y}} \int_{0}^{L_{y}} d y n, \quad v_{0}=\frac{1}{L_{y}} \int_{0}^{L_{y}} d y \frac{\partial \phi}{\partial x},
$$

where $L_{y}$ the poloidal periodicity length. Similarly, we define the spatial fluctuation of any field as the deviation from its profile, and denote this by a tilde. Taking the poloidal average of the particle continuity equation gives

$$
\frac{\partial n_{0}}{\partial t}+\frac{\partial \Gamma_{0}}{\partial x}=S_{n}+D_{n} \frac{\partial^{2} n_{0}}{\partial x^{2}}-\sigma_{n}\left(n_{0}-1\right)
$$

where we have defined the profile of the convective particle flux

$$
\Gamma_{0}=-\frac{1}{L_{y}} \int_{0}^{L_{y}} d y \frac{n}{B} \frac{\partial \phi}{\partial y} .
$$

It is thus clear that the average particle density evolves due to turbulent convective transport (represented by $\Gamma_{0}$ ), fueling (represented by the source $S_{n}$ ), collisional diffusion (represented by the diffusivity $D_{n}$ ), and losses along open field lines in the SOL (represented by the linear damping $\left.\sigma_{n}\right)$. We further define the particle confinement in the edge and SOL regions relative to the basic state,

$$
P_{\text {edge }}=\int_{0}^{L_{y}} d y \int_{0}^{x_{l}} d x(n-1), \quad P_{\mathrm{SOL}}=\int_{0}^{L_{y}} d y \int_{x_{l}}^{L_{x}} d x(n-1) .
$$

Since the convective motion vanishes on the radial boundaries, the sum of these two integrals can only change due to the linear source and sink terms in the particle continuity equation. However, 
the radial convective transport allows a fast transport of particles between the edge and SOL regions. In Sec. III these integrals will be used to characterize the global behavior of the turbulent state.

\section{H. Sheared flows and kinetic energy integrals}

As is well known from basic studies of thermal convection, fluids confined in geometries with periodic directions may generate differential rotation through tilted convection cells. ${ }^{56-58}$ This poloidally mean flow is linearly damped by collisional dissipation but may be non-linearly driven by the fluctuating motions. Indeed, averaging Eq. (20c) over the periodic direction and neglecting the spatial dependence of the sheath damping coefficient we get an equation for the poloidal flow,

$$
\frac{\partial v_{0}}{\partial t}+\frac{\partial}{\partial x}\left(\widetilde{v}_{x} \widetilde{v}_{y}\right)_{0}=D_{\Omega} \frac{\partial^{2} v_{0}}{\partial x^{2}}-\sigma_{\Omega} v_{0}
$$

where $D_{\Omega}$ is the kinematic viscosity. The last term on the left hand side shows that a radial inhomogeneity in the off-diagonal components of the Reynolds stress tensor may provide a source of mean flows. Further integrating over the radial domain, it is clear that the net circulation of any finite system is unaffected by this mechanism, reflecting the absence of an external torque. Hence the ensuing mean flows are intrinsically sheared, corresponding to differential rotation. It is convenient to separate the kinetic energy into two components comprised by convective motions and sheared poloidal flows defined respectively by

$$
K=\int d \mathbf{x} \frac{1}{2}\left(\nabla_{\perp} \widetilde{\phi}\right)^{2}, \quad U=\int d \mathbf{x} \frac{1}{2} v_{0}^{2},
$$

From the vorticity equation (20c) we readily find the evolution of these integrals,

$$
\begin{aligned}
& \frac{d K}{d t}=\zeta \int d \mathbf{x} n T \widetilde{v}_{x}-\int d \mathbf{x} v_{0} \frac{\partial}{\partial x}\left(\widetilde{v}_{x} \widetilde{v}_{y}\right)_{0}-\int d \mathbf{x} \widetilde{\phi} \Lambda_{\Omega}, \\
& \frac{d U}{d t}=\int d \mathbf{x} v_{0} \frac{\partial}{\partial x}\left(\widetilde{v}_{x} \widetilde{v}_{y}\right)_{0}-\int d \mathbf{x} \phi_{0} \Lambda_{\Omega} .
\end{aligned}
$$

The first term on the right hand side of Eq. (29a) shows the fluctuation drive due to radially outwards transport of thermal energy, while the second term reveals the conservative transfer of kinetic energy from fluctuating motions to the sheared flows in Eq. (29b). The last term in either equation describes linear damping due to collisional dissipation and sheath damping. More detailed discussions on convective turbulence with self-sustained sheared flows is given in Refs. 5658. 


\section{SIMULATION RESULTS}

In this section we present results and analysis of two-dimensional simulations of the interchange model discussed in the preceding section. To this end we employ a finite difference method with a symmetry, energy and enstrophy conserving discretization of the non-linear advection terms and an explicit third order stiffly stable time integrator with diffusive terms treated implicitly using operator splitting. ${ }^{65}$ The goal is a detailed comparison with experimental measurements by statistical analysis of probe data obtained from turbulence simulations.

\section{A. Global dynamics and profiles}

Here we present results from a long run simulation with parameters $I=10^{-3}, \zeta=5 \times 10^{-4}, \varepsilon=$ $0.25, q=3$, and all collisional diffusivities are set to $10^{-2}$. The numerical grid consists of $512 \times$ 256 nodes in the radial versus poloidal direction, respectively. Let us begin by describing the global behavior of a typical turbulent state. Presented in Fig. 2 is the evolution of the kinetic energy in the fluctuating motions and in the sheared poloidal flows. The intensity of the fluctuating motions show irregular oscillations with pronounced bursts. Whenever this fluctuation level is sufficiently large, there is a rapid growth of the energy in the sheared poloidal flows. This is followed by a suppressed level of fluctuation kinetic energy while the flow energy is slowly damped, resulting in quasi-periodic relaxation oscillations of the latter. Such a dynamical regulation results from the kinetic energy transfer directed from the fluctuating motions to the sheared flows and the viscous damping of the latter on a slow time scale. A detailed discussion of this predator-prey like behavior from the point of view of energetics was given in Refs. 57 and 58. Typical for these convectionshear flow systems, the kinetic energy in the mean flows is much larger than that of the fluctuating motions except during turbulence bursts. To further support the above interpretation we present in Fig. 3 the evolution of the kinetic energy transfer rate and the turbulence energy drive, defined respectively by

$$
F_{v}=\int d \mathbf{x} \frac{\partial v_{0}}{\partial x}\left(\widetilde{v}_{x} \widetilde{v}_{y}\right)_{0}, \quad F_{p}=\zeta \int d \mathbf{x} n T \widetilde{v}_{x}
$$

Note that the latter is just a measure of the radial thermal energy flux. Both of these integral quantities show large peaks corresponding to the bursts in the energy of the fluctuating motions. Moreover, they clearly demonstrate a uni-directional energy transfer from the confined thermal energy to the kinetic energy of the fluctuation motions, and from the fluctuating motions to the 
sheared poloidal flows.

Associated to the bursting in the kinetic energy of the fluctuating motions is a drastic change in the particle and heat confinement. In Fig. 4 we present the particle confinement in the edge and the SOL regions. It is clearly seen that correlated to the large peaks in the kinetic energy of the fluctuating motions is a rapid decay of the confinement in the edge region and a corresponding increase of particles in the SOL. Figure 4 thus reveals a bursty ejection of particles from the edge to the SOL. It is of interest to note that the resulting relaxation oscillations of the edge plasma confinement were also described by the zero-dimensional modeling in Ref. 58 . We also remark that the heat confinement shows a behavior similar to that presented in Fig. 4 but with lower amplitudes due to the stronger sheath damping in the region of open field lines. ${ }^{60}$

The evolution of the particle confinement in Fig. 4 indicates that the largest value of the particle density is in the edge region. Indeed, the time-averaged profiles of the particle density $\bar{n}_{0}$ and the temperature $\bar{T}_{0}$ shown in Fig. 5 reveal strong gradients in the edge region while the profiles are nearly flat in the SOL. The confinement variations seen in Fig. 4 are due to significant variations in these profiles from their time-averaged values. ${ }^{60}$ The vertical bars also shown in Fig. 5 indicate the standard deviation and the extremal values of the local particle density as measured by the probes $P_{i}$ indicated in Fig. 1. Order unit perturbations to the time-averaged profiles may occur locally. However, in the far SOL strong sheath damping and the somewhat artificial boundary conditions prevent significant fluctuations.

In Fig. 6 we further present the time-averaged profile of the total radial particle flux $\bar{F}_{0}$ as well as its convective contribution $\bar{\Gamma}_{0}$. Clearly, the diffusive radial particle flux, being the difference between these curves, is negligible in the SOL. In the edge region the total radial flux is given by the input rate of the particle source, here $10^{-3}$, while the particle loss along open field lines gives rise to a decaying particle flux profile in the SOL region. Such a separation of the particle and heat fluxes into their radial and parallel components is of great interest for transport modeling of the SOL but a qualitative prediction unfortunately requires an exact description of the full parallel particle motions, including that of the sheath dynamics, and is beyond the scope of this work.

Finally, in Fig. 7 we also show the time-averaged radial profiles of the poloidal flow $\bar{v}_{0}$ and the vorticity $\bar{\Omega}_{0}$. The mean flow has a peak at the left boundary with a value of one quarter of the acoustic speed and reverses direction just inside the LCFS. The transit time associated with the fast poloidal rotation in the edge region introduces a temporal periodicity on the local probe signals when the correlation time is of order of or larger than this transit time. Note that for the 
simple fluid model used here there is no preferred sign of the net vorticity, which thus follows from an initial seed or round-off errors in the simulations.

\section{B. Two-dimensional structures}

We next report on some details regarding the spatial structure of the dependent variables from the two-dimensional turbulence simulations. As shown in Fig. 2, the global fluctuation level appears in bursts separated by relatively quiet periods. In Fig. 8 we present the spatial structure of the particle density, temperature, electrostatic potential and vorticity field in a typical quiet period, at time $t=1.064 \times 10^{6}$, and during a burst in the global fluctuation level, at time $t=1.072 \times 10^{6}$. For the electrostatic potential we have subtracted the poloidal average which would otherwise completely dominate the contour lines. The vertical lines indicate the transition from the edge to the SOL, and the stars show the location of the probes $P_{i}$ from which data time series are recorded.

In the quiet period there are almost no spatial fluctuations and the plasma is nearly homogeneous and isothermal in the SOL. Strong radial gradients are evident in the particle density and the temperature fields in the edge region. At the time of bursting in the fluctuation energy we observe strong perturbations in all dependent variables, with prominent structures in the SOL. Typical for turbulent convection, there are plume structures in the particle density and temperature fields which have a mushroom-like shape. Not surprisingly, this was also observed in the idealized blob simulations of a simple convection model in Ref. 42. As the temperature field is more heavily damped in the SOL, its contour plot shows smaller amplitudes than the particle density. Associated to these plume structures are dipoles in the electrostatic potential and vorticity fields. The corresponding electric charge polarization is consistent with that due to the compressible diamagnetic current in the inhomogeneous magnetic field, reflecting vertical magnetic guiding center drifts within the blob structures. ${ }^{40-42}$

Snapshots from the time evolution of the particle density during the burst discussed above are shown in Fig. 9. Here we clearly see the radial propagation of a blob structure formed close to the LCFS. Initially there is a coherent blob propagating predominantly with the local poloidal electric drift. The magnetic guiding center drifts cause an electric charge polarization and thereby a radial velocity component. This standard picture of blob propagation is then followed by a strongly nonlinear evolution whereby the structure develops a mushroom-like shape followed by a pronounced tail. The blob fragments into two weakly connected entities corresponding to a separation of the 
dipolar structure of the electrostatic potential and the vorticity fields seen in the last panel of Fig. 8 . Further on the leading front of the initial blob structure completely vanishes. We also see in the fourth panel that the primary blob structure is followed by a secondary one which has a weaker amplitude and thus a smaller radial velocity component. From the non-linear evolution of these structures it is apparent that the time-series recorded at fixed probe positions will have a highly irregular shape and may also be influenced by the finite poloidal periodicity length.

\section{Distributions and conditional structures}

The raw signal of the particle density fluctuations measured by the probes $P_{1}$ to $P_{6}$ are presented in Fig. 10. We first note that the particle density at the probe $P_{1}$ in the edge region shows chaotic oscillations which appears as symmetric about the mean value. On the other hand, all the other probe signals reveal predominantly positive fluctuations generally occurring with a fast rise followed by damped irregular oscillations. It is also clear that some events are correlated through all the probe signals, indicating a radial propagation. In the following we will present results from statistical analyzes of these probe data which indeed confirm such long-range transient transport events.

The rescaled PDFs of locally measured particle density fluctuations shown in Fig. 11 reveal positively skewed and flattened distributions in the SOL, reflecting the high probability for large fluctuations as is evidently seen from the raw time series presented in Fig. 10. The particle density PDF is close to a normal distribution in the edge region but possesses a gradually fatter tail radially outwards. Moreover, the PDFs have a self-similar functional form in the SOL with the distributions rescaled with the standard deviation $n_{\mathrm{rms}}$ and shifted with the average value $\bar{n}$ fall on top of each other. We note that the nearly normal distribution in the edge region and the positively skewed and flat distributions in the SOL are qualitatively similar to that observed in numerous laboratory experiments. ${ }^{11-22}$

In Fig. 12 we present the conditionally averaged particle density signals for the seven different probes shown in Fig. 1. For each probe position the local condition $n-\bar{n}>4 n_{\mathrm{rms}}$ is used to select the conditional sub-records, with $\bar{n}$ and $n_{\text {rms }}$ the time-average and standard deviation of the density signal at each individual probe position. There are several prominent features in Fig. 12 which are similar to that measured in laboratory experiments. ${ }^{14-22}$ First, there is evidently a coherent wave form at all probe positions with the maximum amplitude decaying throughout the SOL. Second, 
the conditional structures show a significant elevation above the background level. Finally, the conditional signal has a sharp rise followed by a relatively slow decay. These results are consistent with radially propagating structures whose amplitudes gradually diminish due to particle losses along open field lines.

Using the same condition on the particle density fluctuations, we show in Fig. 13 the conditionally averaged wave form of the radial electric drift. Again, on the innermost probes we observe a sharp rise followed by a slow decay, and a weak delay for the peak on the outer probe positions. Note that the peak value of the radial conditional velocity is $5 \%$ of the acoustic speed. The periodic oscillation at probe $P_{1}$ is an artifact of the relatively short poloidal periodicity length together with the strong poloidal flow in the edge region, and is not observed in experimental measurements. Similarly, the rather wide conditional wave form recorded on probe $P_{2}$ is related to the vanishing of the poloidal flow at the LCFS. Otherwise, these results are in good agreement with numerous laboratory investigations. ${ }^{11-22}$

\section{Transport statistics}

A plot of the rescaled PDFs of the radial turbulent particle flux $(n-\bar{n}) v_{x}$ recorded by the probes $P_{i}$ are shown in Fig. 14. Typical for turbulent transport problems, the radial flux PDF exhibits an exponential tail for large values. ${ }^{66-68}$ While negative flux events do occur, the large positive bursts are dominant, leading to an extended tail towards radially outwards particle fluxes. The rescaled PDFs nearly fall on top of each other for all radial positions, comprising the edge region as well as the SOL, thus indicating the possibility of a self-similar functional form for the turbulent particle flux at the plasma boundary.

We further present a conditional average analysis of the poloidally averaged radial turbulent particle flux $\Gamma_{0}$ at the radial position corresponding to probe $P_{3}$ in Fig. 1. As in the previous analysis we use a signal of $6 \times 10^{5}$ points with a sampling time of 2.5. A standard conditional average is performed according to the amplitude condition $\Gamma_{0}-\bar{\Gamma}_{0}>\alpha \Gamma_{0 \text { rms. }}$. In Fig. 15 we present the summed conditional particle flux $\Gamma_{\alpha}$ relative to the total accumulated flux $\Gamma_{\Sigma}$ as a function of the condition $\alpha$. The conditional sub-records consist of 600 points, or 1500 time units, which should be compared to the duration of the conditional wave forms shown in Fig. 12. Clearly, for negative conditions the sub-records comprise the full time series and the contribution of the conditional flux equals the time-average of the full time series. Increasing the condition 
parameter $\alpha$, more and more of the original time series is excluded and so the relative contribution of the conditional flux events decreases. However, three measures of the intermittency of the turbulent transport is readily apparent. First, $80 \%$ of the total flux is due to conditional flux events larger than the flux standard deviation. Second, $50 \%$ of the total flux is due to conditional flux events with an amplitude larger than 2.5 times the rms value, and third, $20 \%$ of the flux is due to conditional events larger than 5 times the standard deviation of the convective particle flux. This is in quantitative agreement with similar analysis performed on experimental data. ${ }^{17,18}$

Also shown in Fig. 15 is the count number $N(\alpha)$ of conditional transport events as a function of the condition $\alpha$ relative to the maximum number of conditional sub-records, $N_{\Sigma}=10^{3}$. This curve shows that while half of the total flux is due to conditional flux events with peak amplitudes larger than 2.5 times the standard deviation, these events fill only $20 \%$ of the full time record. Similarly, the flux events with the condition $\alpha \geq 5$ comprise $20 \%$ of the total flux but their duration is less than one tenth of the full time series. These numbers serve to manifest the intrinsic intermittent nature of the turbulent convection in the plasma boundary, and is again in excellent agreement with experimental measurements. ${ }^{17,18}$ We also note that similar results were found in the seminal work done on the Ris $\varnothing$ Q-machine. ${ }^{29}$

As mentioned previously in the discussion of Fig. 2, the turbulence appears in bursts at quasiperiodic intervals given by the viscous dissipation rate of the sheared poloidal flows. A measure of this repetition frequency is readily given by the waiting time statistics for conditional transport events. In Fig. 16 we present the histogram for the waiting time $\triangle t$ between successive conditional events in the poloidally averaged radial convective particle flux. As in the above analysis a subrecord length of 600 points or 1500 time units is used, and the averaging condition $\Gamma_{0}-\bar{\Gamma}_{0}>$ $4 \Gamma_{0 \mathrm{rms}}$ yields merely 66 events. Nevertheless, the waiting time statistics in Fig. 16 clearly reveals a peak value of the probability at a time lag of $10^{4}$. There is, however, a pronounced tail towards larger waiting times, resulting in a mean value of $2 \times 10^{4}$. Similar distributions of the waiting time statistics for ion saturation current measurements in the SOL plasma of several experimental configurations have been reported recently. ${ }^{12,14,15}$ While the physical mechanism underlying the quasi-periodicity in the present simulations may differ from that of the actual experiments, we note that profile relaxations in general may lead to a dynamical regulation involving bursting in the global fluctuation level. ${ }^{58}$ 


\section{DISCUSSION AND CONCLUSIONS}

In the first part of this paper we have derived a set of reduced fluid equations suitable for the description of electrostatic interchange motions in non-uniformly magnetized plasmas. Particular attention has been given to the particle and energy conservation properties, and to retain the self-consistent evolution of the full profiles of the dependent variables. The need for the latter is clearly indicated by the experimental observations of coherent plasma blobs with amplitudes significantly exceeding that of the background plasma. In order to allow statistical averages we have considered a two-dimensional slab model accounting for the three-dimensional structure of the blob structures by including loss terms in an effective SOL region to model the transport along open magnetic field lines. In the second part of this work we have presented results from numerical solutions of this model for geometry and parameters relevant for the edge and SOL of magnetically confined plasmas. As shown in the preceding section, detailed analysis of the simulation data yields favorable comparisons with the many recent experimental measurements. ${ }^{2-28}$

During the last couple of years there has emerged a theory of radial blob propagation based on the vertical vorticity polarization due to magnetic guiding center drifts in non-uniformly magnetized plasmas. ${ }^{40,41}$ Numerical simulations of isolated blob structures, ${ }^{42-44}$ as well as the more comprehensive results presented here, confirm that the vorticity polarization mechanism leads to radial self-advection of coherent structures, one example presented in Fig. 9. In this work it is further shown that plasma blobs non-linearly develop a mushroom-like shape, usually with a pronounced tail. It is of interest to note that such wakes are also visible in the fast camera imaging of

blobs in the SOL. ${ }^{28}$ During the non-linear evolution there is a fragmentation of the blob structure with residual plasma trapped in separate vortices of opposite polarity. The internal differential rotation prevents a secondary polarization of vorticity, and thus brings the radial propagation to a halt.

Careful statistical analysis of simulation data recorded at fixed probe locations as shown in Fig. 1 yields numerous results in excellent agreement with experimental measurements. In particular, we observe that the PDF of the particle density fluctuations is normal in the edge region but possess increasingly larger skewness and flatness factors outwards in the SOL. This reflects the frequent appearance of large bursts in the time records corresponding to blobs of excess particles and heat as compared to the ambient plasma. Moreover, the rescaled PDFs indicate a self-similar functional form in the SOL. The conditionally averaged structure of the particle density and tem- 
perature show the typical asymmetric wave form with a sharply rising front and a trailing wake. Associated with this front in the thermodynamic fields is a radially outward electric drift as demonstrated by the cross-conditional radial velocity shown in Fig. 13, revealing a maximum value of 5\% of the acoustic speed. The amplitude of these coherent structures decay as they propagate though the SOL partly due to the effective sheath damping and partly due to non-linear fragmentation and mixing. The formation of blob structures is demonstrated to be related to profile relaxations in the strongly turbulent edge region, a process that is triggered in a quasi-periodic manner by a global regulation due to self-sustained sheared poloidal flows. ${ }^{56-60}$

Despite the favorable agreement between the results presented here and the many recent experimental measurements of intermittent SOL transport, it is worth emphasizing that the model used here is far from a complete description of the plasma boundary region. In particular, we have neglected magnetic shear and parallel electric currents in the edge plasma as well as impurity and neutral particles. Moreover, the parallel transport along open magnetic field lines has been modeled as a simple linear damping of all dependent variables. The competition between parallel and cross-field transport leads to the time-averaged profile shown in Fig. 6 and determines the SOL width. However, an accurate prediction of such an important measure of plasma and heat transport in the SOL requires a more careful treatment of the sheath physics and is beyond the scope of this work. What is clear from the present results is that a successful description of SOL turbulence must account for the full profile evolution and thus relax the thin layer approximation inherent in reduced fluid models used presently.

\section{Acknowledgments}

This work was supported by the Danish Center for Scientific Computing through Grants No. CPU-1101-08 and CPU-1002-17. O. E. G. was supported with financial subvention from the Research Council of Norway. Discussions with V. Antoni, W. Fundamenski, O. Grulke, R. Schneider and S. J. Zweben are gratefully acknowledged.

\footnotetext{
* Electronic address: odd.erik.garcia@risoe.dk
} 
1 P. C. Stangeby, The plasma boundary of magnetic fusion devices (Institute of Physics Publishing, Bristol, 2000).

2 S. J. Zweben, Phys. Fluids 28, 974 (1985).

3 S. J. Zweben and R. W. Gould, Nucl. Fusion 25, 171 (1985).

4 M. Endler, J. Nucl. Mater. 266-269, 84 (1999).

5 M. Endler, L. Giannone, K. McCormick, H. Niedermeyer, A. Rudyj, G. Theimer, N. Tsois, S. Zoletnik, and the ASDEX Team, Physica Scripta 51, 610 (1995).

6 E. Sánchez, C. Hidalgo, D. López-Bruna, I. Garcia-Cortés, R. Balbin, M. A. Pedrosa, B. van Milligen, C. Riccardi, G. Chiodini, J. Bleuel, M. Endler, B. A. Carreras, and D. E. Newman, Phys. Plasmas 7, 1408 (2000).

7 O. Grulke, T. Klinger, M. Endler, A. Piel, and the W7-AS Team, Phys. Plasmas 8, 5171 (2001).

8 J. Bleuel, M. Endler, H. Niedermeyer, M. Schubert, H. Thomsen, and the WS7-AS Team, New J. Phys. 4, 38 (2002).

9 V. Antoni, H. Bergsåker, G. Seranni, M. Spolaore, N. Vianello, R. Cavazzana, G. Regnoli, E. Spada, E. Martines, M. Bagatin, and J. R. Drake, J. Nucl. Mater. 313-316, 972 (2003).

10 M. Spolaore, V. Antoni, E. Spada, H. Bergsåker, R. Cavazzana, J. R. Drake, E. Martines, G. Regnoli, G. Serianni, and N. Vianello, Phys. Rev. Lett. 93, 215003 (2004).

11 F. Sattin, N. Vianello, and M. Valisa, Phys. Plasmas 11, 5032 (2004).

12 G. Y. Antar, Phys. Plasmas 10, 3629 (2003).

13 G. Y. Antar, S. I. Krasheninnikov, P. Devynck, R. P. Doerner, E. M. Hollmann, J. A. Boedo, S. C. Luckhardt, and R. W. Conn Phys. Rev. Lett. 87, 065001 (2001).

14 G. Y. Antar, G. Counsell, Y. Yu, B. LaBombard, and P. Devynck, Phys. Plasmas 10, 419 (2003).

15 G. Y. Antar, P. Devynck, X. Garbet, and S. C. Luckhardt, Phys. Plasmas 8, 1612 (2001).

16 J. P. Graves, J. Horacek, R. A. Pitts, and K. I. Hopcraft, Plasma Phys. Controlled Fusion 47, L1 (2005).

17 G. S. Kirnev, V. P. Budaev, S. A. Grashin, E. V. Gerasimov, and L. N. Khimchenko, Plasma Phys. Controlled Fusion 46, 621 (2004).

18 J. A. Boedo, D. Rudakov, R. Moyer, S. Krasheninnikov, D. Whyte, G. McKee, G. Tynan, M. Schaffer, P. Stangeby, P. West, S. Allen, T. Evans, R. Fonck, E. Hollmann, A. Leonard, A. Mahdavi, G. Porter, M. Tillack, and G. Antar, Phys. Plasmas 8, 4826 (2001).

19 J. A. Boedo, D. L. Rudakov, R. J. Colchin, R. A. Moyer, S. Krasheninnikov, D. G. Whyte, G. R. McKee, G. Porter, M. J. Schaffer, P. C. Stangeby, W. P. West, S. L. Allen, and A. W. Leonard, J. Nucl. Mater. 
313-316, 813 (2003).

20 J. A. Boedo, D. L. Rudakov, R. A. Moyer, G. R. McKee, R. J. Colchin, M. J. Schaffer, P. G. Stangeby, W. P. West, S. L. Allen, T. E. Evans, R. J. Fonck, E. M. Hollmann, S. Krasheninnikov, A. W. Leonard, W. Nevins, M. A. Mahdavi, G. D. Porter, G. R. Tynan, D. G. Whyte, and X. Xu, Phys. Plasmas 10, 1670 (2003).

21 D. Rudakov, J. A. Boedo, R. A. Moyer, S. Krasheninnikov, A. W. Leonard, M. A. Mahdavi, G. R. McKee, G. D. Porter, P. C. Stangeby, J. G. Watkins, W. P. West, D. G. Whyte, and G. Antar, Plasma Phys. Controlled Fusion 44, 717 (2002).

22 M. V. A. P. Heller, Z. A. Brasilio, I. L. Caldas, J. Stöckel, and J. Petrzilka, Phys. Plasmas 6, 846 (1999).

23 M. Endler, H. Niedermeyer, L. Giannone, E. Holzhauer, A. Rudyj, G. Theimer, N. Tsois, and the ASDEX Team, Nucl. Fusion 35, 1307 (1995).

24 A. K. Singh, J. Morelli, T. Asai, and A. Hirose, Phys. Plasmas 10, 3451 (2003).

25 R. J. Maqueda, G. A. Wurden, S. J. Zweben, L. Roquemore, H. Kugel, D. Johnson, S. Kaye, S. Sabbagh, and R. Maingi, Rev. Sci. Instr. 72, 931 (2001).

26 S. J. Zweben, D. P. Stotler, J. L. Terry, B. LaBombard, M. Greenwald, M. Muterspaugh, C. S. Pitcher, K. Hallatschek, R. J. Maqueda, B. Rogers, J. L. Lowrance, V. J. Mastrocola, and G. F. Renda, Phys. Plasmas 9, 1981 (2002).

27 J. L. Terry, S. J. Zweben, K. Hallatschek, B. LaBombard, R. J. Maqueda, B. Bai, C. J. Boswell, M. Greenwald, D. Kopon, W. M. Nevins, C. S. Pitcher, B. N. Rogers, D. P. Stotler, and X. Q. Xu, Phys. Plasmas 10, 1739 (2003).

28 S. J. Zweben, R. J. Maqueda, D. P. Stotler, A. Keesee, J. Boedo, C. E. Bush, S. M. Kaye, B. LeBlanc, J. L. Lowrance, V. J. Mastrocola, R. Maingi, N. Nishino, G. Renda, D. W. Swain, J. B. Wilgen and the NSTX Team, Nucl. Fusion 44, 134 (2004).

29 T. Huld, A. H. Nielsen, H. L. Pécseli, and J. Juul Rasmussen, Phys. Fluids B 3, 1609 (1991).

30 A. H. Nielsen, H. L. Pécseli, and J. Juul Rasmussen, Phys. Plasmas 3, 1530 (1996).

31 F. J. Øynes, H. L. Pécseli, and K. Rypdal, Phys. Rev. Lett. 75, 81 (1995).

32 F. J. Øynes, O.-M. Olsen, H. L. Pécseli, Å. Fredriksen, and K. Rypdal, Phys. Rev. E 57, 2242 (1998).

33 K. Rypdal, O. E. Garcia, and J.-V. Paulsen, Phys. Rev. Lett. 79, 1857 (1997).

34 O. Grulke, F. Greiner, T. Klinger, and A. Piel, Plasma Phys. Controlled Fusion 43, 525 (2001).

35 B. Gonçalves, C. Hidalgo, M. A. Pedrosa, C. Silva, R. Balbín, K. Erents, M. Hron, A. Loarte, and G. Matthews, Plasma Phys. Controlled Fusion 45, 1627 (2003). 
36 T. Eich, A. Herrmann, J. Neuhauser, and ASDEX Upgrage Team, Phys. Rev. Lett. 91, 195003 (2003).

37 W. Fundamenski, W. Sailer, and JET EFDA contributors, Plasma Phys. Controlled Fusion 46, 233 (2004).

38 A. Kirk, H. R. Wilson, G. F. Counsell, R. Akers, E. Arends, S. C. Cowley, J. Dowling, B. Lloyd, M. Price, M. Walsh, and MAST Team, Phys. Rev. Lett. 92, 245002 (2004).

39 M. Endler, I. García-Cortés, C. Hidalgo, G. F. Matthews, ASDEX Team, and JET Team, Plasma Phys. Controlled Fusion 47, 219 (2005).

40 S. I. Krashninnikov, Phys. Lett. A 283, 368 (2001).

41 D. A. D’Ippolito, J. R. Myra, and S. I. Krashninnikov, Phys. Plasmas 9, 222 (2002).

42 N. Bian, S. Benkadda, J.-V. Paulsen, and O. E. Garcia, Phys. Plasmas 10, 671 (2003).

43 G. Q. Yu and S. I. Krashninnikov, Phys. Plasmas 10, 4413 (2003).

44 D. A. D’Ippolito, J. R. Myra, S. I. Krashninnikov, G. Q. Yu, and A. Y. Pigarov, Contrib. Plasma Phys. 44, 205 (2004).

45 J. R. Myra, D. A. D’Ippolito, S. I. Krashninnikov, and G. Q. Yu, Phys. Plasmas 11, 4267 (2004).

46 D. A. D’Ippolito, J. R. Myra, D. A. Russell, and G. Q. Yu, Phys. Plasmas 11, 4603 (2004).

47 O. Pogutse, W. Kerner, V. Gribkov, S. Bazdenkov, and M. Osipenko, Plasma Phys. Controlled Fusion 36, 1963 (1994).

48 S. Benkadda, X. Garbet, and A. Verga, Contrib. Plasma Phys. 34, 247 (1994).

49 S. Benkadda, T. Dudok de Wit, A. Verga, A. Sen, ASDEX team, and X. Garbet, Phys. Rev. Lett. 73, 3403 (1994).

50 Y. Sarazin and Ph. Ghendrih, Phys. Plasmas 5, 4214 (1998).

$51 \mathrm{Ph}$. Ghendrih, Y. Sarazin, G. Attuel, S. Benkadda, P. Beyer, G. Falchetto, C. Figarella, X. Garbet, V. Grandgirard, and M. Ottaviani, Nucl. Fusion 43, 1013 (2003).

52 Y. Sarazin, Ph. Ghendrih, G. Attuel, C. Clément, X. Garbet, V. Grandgirard, M. Ottaviani, S. Benkadda, P. Beyer, N. Bian, and C. Figarella, J. Nucl. Mater. 313-316, 796 (2003).

53 N. Bisai, A. Das, S. Deshpande, R. Jha, P. Kaw, A. Sen, and R. Singh, Phys. Plasmas 11, 4018 (2004).

54 X. Q. Xu, W. M. Nevins, R. H. Cohen, J. R. Myra, and P. B. Snyder, New J. Phys. 4, 53 (2002).

55 D. A. Russell, D. A. D’Ippolito, J. R. Myra, W. M. Nevins, and X. Q. Xu, Phys. Rev. Lett. 93, 265001 (2004).

56 O. E. Garcia, N. H. Bian, J.-V. Paulsen, S. Benkadda, and K. Rypdal, Plasma Phys. Controlled Fusion 45, 919 (2003). 
57 O. E. Garcia and N. H. Bian, Phys. Rev. E 68, 047301 (2003).

58 N. H. Bian and O. E. Garcia, Phys. Plasmas 10, 4696 (2003).

59 V. Naulin, J. Juul Rasmussen, and J. Nycander, Phys. Plasmas 10, 1075 (2003).

60 O. E. Garcia, V. Naulin, A. H. Nielsen, and J. Juul Rasmussen, Phys. Rev. Lett. 92, 165003 (2004).

61 O. E. Garcia, J. Plasma Phys. 65, 81 (2001).

62 O. E. Garcia, Europ. J. Phys. 24, 313 (2003).

63 J. F. Drake and T. M. Antonsen Jr., Phys. Fluids 27, 898 (1983).

64 M. Yagi and W. Horton, Phys. Plasmas 1, 2135 (1994).

65 V. Naulin and A. H. Nielsen, SIAM J. Sci. Comp. 25, 104 (2003).

66 B. A. Carreras, C. Hidalgo, E. Sánchez, M. A. Pedrosa, R. Balbín, I. García-Cortés, B. van Milligen, D. E. Newman, and V. E. Lynch, Phys. Plasmas 3, 2664 (1996).

67 C. Hidalgo, B. Gonçalves, M. A. Pedrosa, J. Castellano, K. Erents, A. L. Fraguas, M. Hron, J. A. Jiménez, G. F. Matthews, B. van Milligen, and C. Silva, Plasma Phys. Controlled Fusion 44, 1557 (2002).

68 V. Naulin, O. E. Garcia, A. H. Nielsen, and J. J. Rasmussen, Phys. Lett. A 321, 355 (2004). 
FIG. 1: (Color online). Setup of the simulation domain showing the forcing region to the left, corresponding to the edge plasma, and the parallel loss region to the right, corresponding to the SOL. Data time series are recorded by the probes $P_{i}$.

FIG. 2: (Color online). Temporal evolution of the kinetic energy in the fluctuating motions, $K$, and in the sheared poloidal flows, $U$.

FIG. 3: (Color online). Temporal evolution of the kinetic energy transfer rate from the fluctuating motions to the sheared flows, $F_{v}$, and the transfer rate from confined thermal energy to fluctuating motions, $F_{p}$.

FIG. 4: (Color online). Temporal evolution of the particle confinement in the edge region, $P_{\text {edge }}$, and in the SOL region, $P_{\mathrm{SOL}}$.

FIG. 5: (Color online). Time averaged profile of the particle density, $\bar{n}_{0}$, and the electron temperature, $\bar{T}_{0}$. The vertical bars indicate the total range of the local particle density fluctuations, $\widetilde{n}$, as well as the root mean square values, $n_{\mathrm{rms}}$, about the average values as recorded by the probes $P_{i}$.

FIG. 6: (Color online). Time averaged profile of the total, $\bar{F}_{0}$, and the convective, $\bar{\Gamma}_{0}$, particle fluxes which decay in the SOL due to particle losses along open magnetic field lines.

FIG. 7: (Color online). Time averaged profiles of the poloidal flow, $\bar{v}_{0}$, and the vorticity of the electric drift, $\bar{\Omega}_{0}$.

FIG. 8: (Color online). From top to bottom, the spatial structure of the particle density, temperature, electrostatic potential and vorticity during a quiet period to the left and during a burst to the right. The poloidal average has been subtracted for the electrostatic potential. The vertical lines indicate the separation between the edge and the SOL regions while the stars show the position of the probes $P_{i}$.

FIG. 9: (Color online). Spatial structure of the particle density showing radial blob propagation during a burst in the global fluctuation level. 
FIG. 10: (Color online). Temporal evolution of the particle density measured by the different probes, starting with $P_{1}$ in the lowest curve and ending with $P_{6}$ in the uppermost curve. Each signals is amplified by a factor two and shifted by unity for illustration.

FIG. 11: (Color online). Rescaled probability distribution functions of the particle density signals measured by the probes $P_{i}$, revealing a normal distribution in the edge and positively skewed and flattened distributions in the SOL.

FIG. 12: (Color online). Conditional average of the particle density signals recorded by the probes $P_{i}$, showing a coherent asymmetric wave form with a gradual decay of the amplitude outwards in the SOL.

FIG. 13: (Color online). Conditional average of the radial velocity signals recorded by the probes $P_{i}$ with the same condition on the particle density and the same linestyle as used for Fig. 12.

FIG. 14: (Color online). Rescaled probability distribution functions of the radial turbulent particle flux $(n-\bar{n}) v_{x}$ measured by the probes $P_{i}$.

FIG. 15: (Color online). The upper curve shows the accumulated conditional particle flux $\Gamma_{\alpha}$ relative to the total flux $\Gamma_{\Sigma}$ at the radial position corresponding to probe $P_{3}$. The flux is averaged over the poloidal direction, and the conditional averaging is defined by $\left\langle\Gamma_{0} \mid \Gamma_{0}-\bar{\Gamma}_{0}>\alpha \Gamma_{0 \text { rms }}\right\rangle$. The lower curve shows the count number $N_{\alpha}$ of conditional flux sub-records relative to the maximum number $N_{\Sigma}$ of sub-records in the full time series.

FIG. 16: (Color online). Histogram of waiting times for conditional events in the poloidally averaged radial particle flux at the radial position corresponding to probe $P_{3}$, revealing the quasi-periodic occurence of transport bursts. 


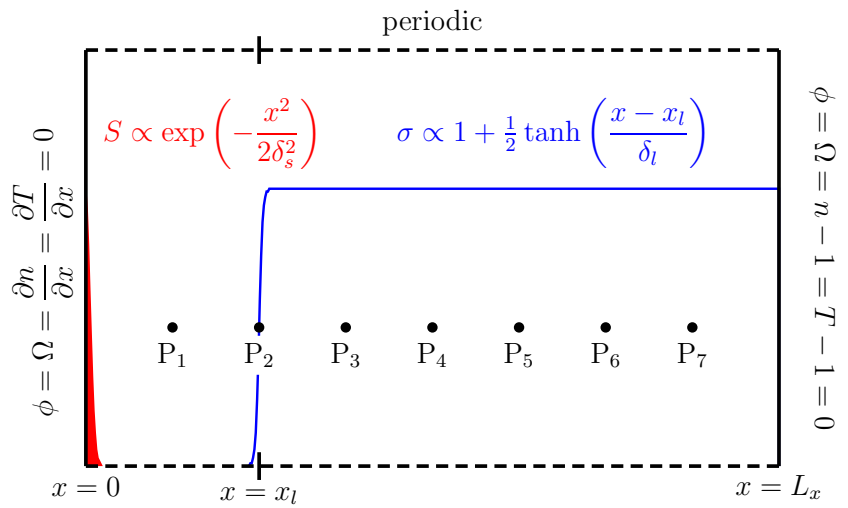

FIG. 1

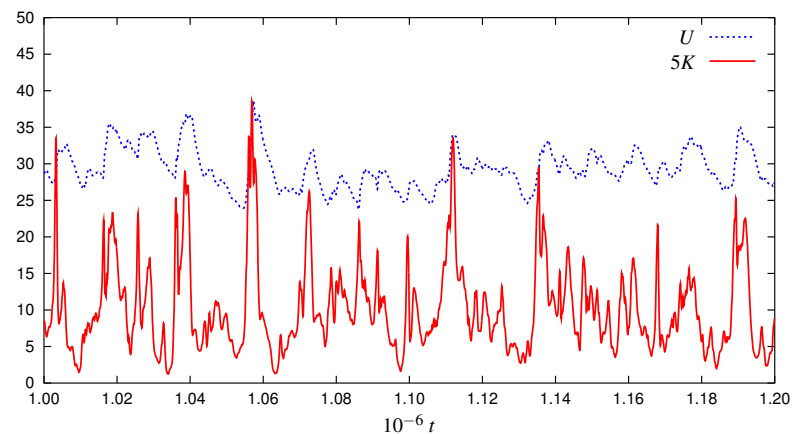

FIG. 2

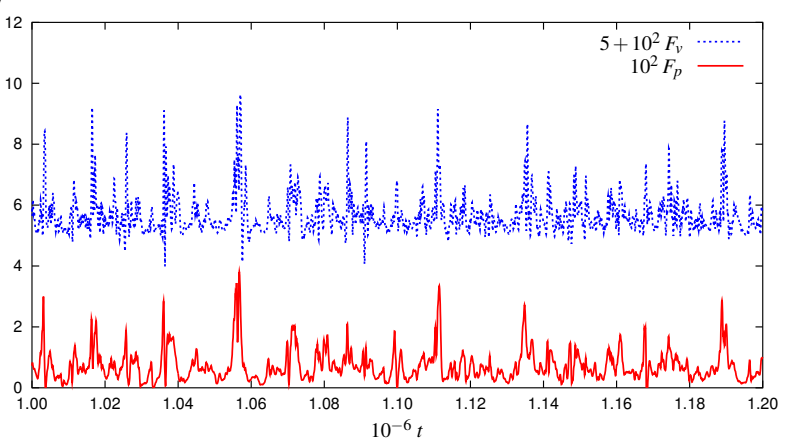

FIG. 3

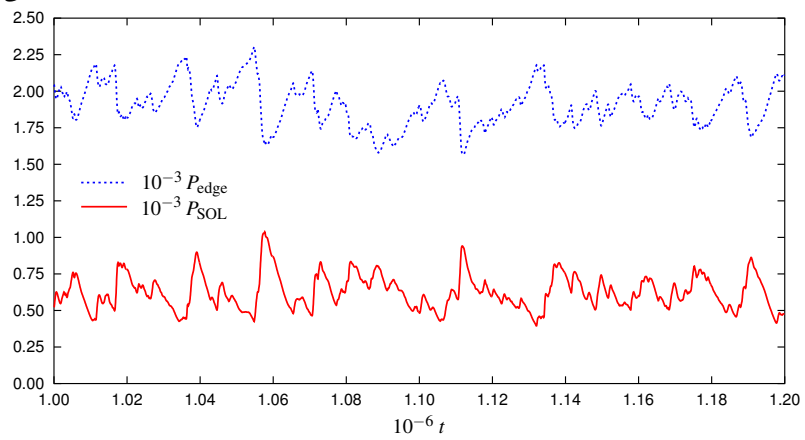

FIG. 4 


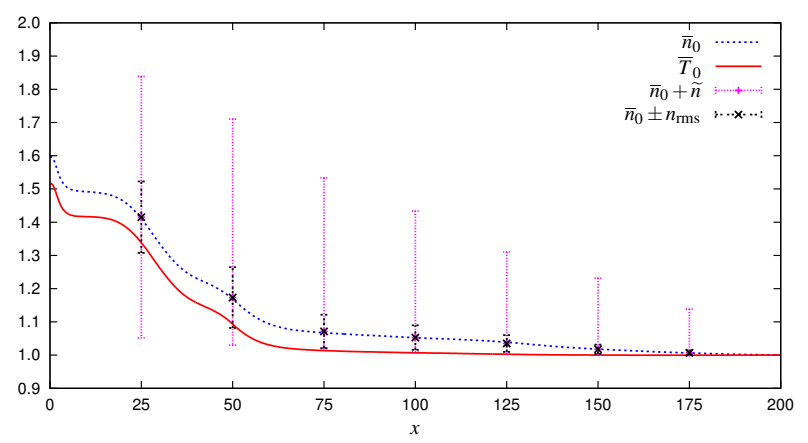

FIG. 5

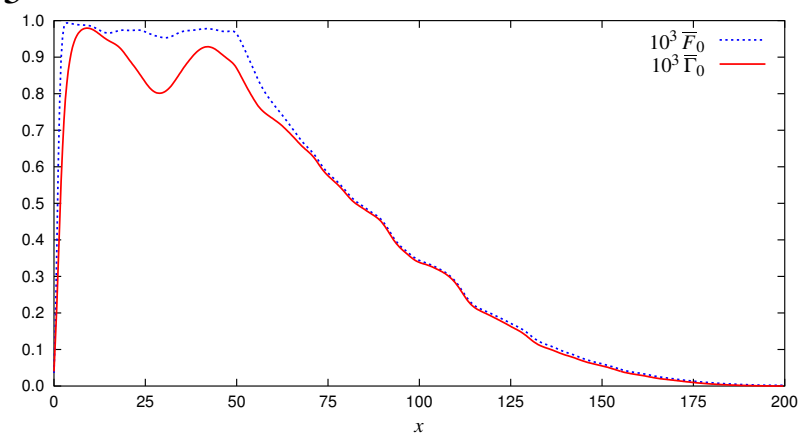

FIG. 6

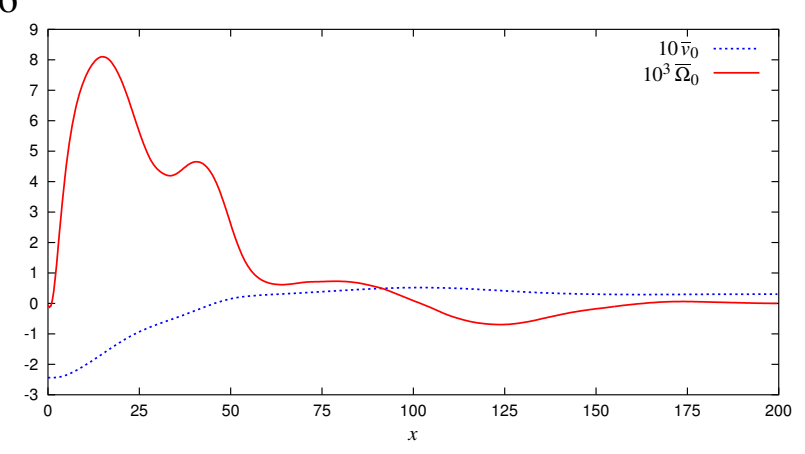

FIG. 7 

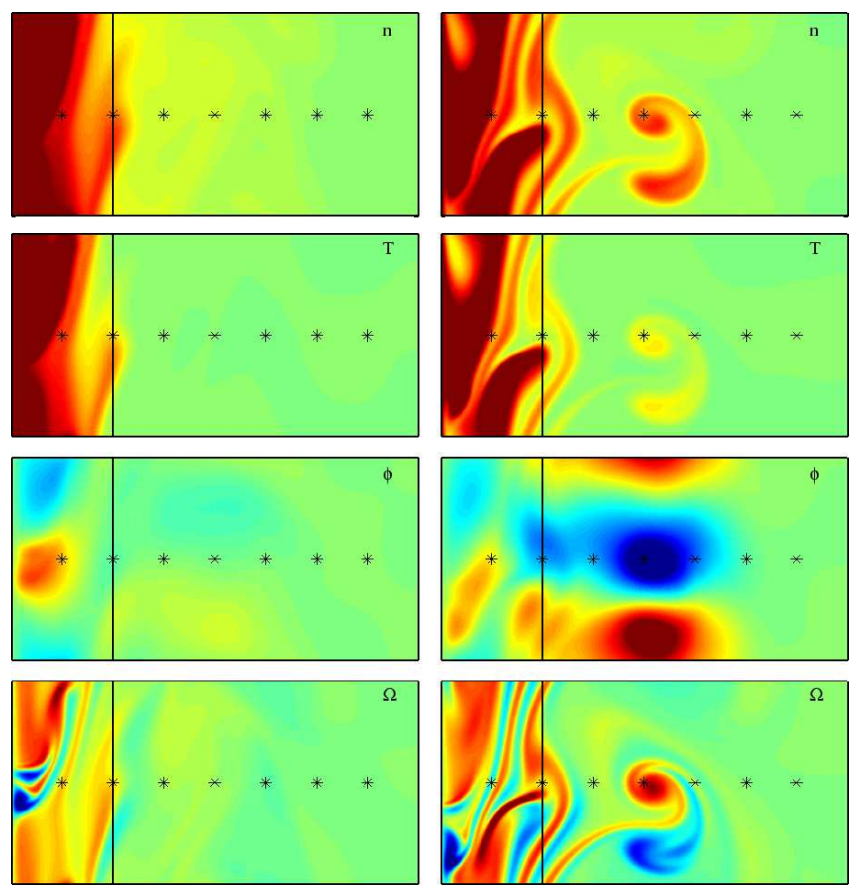

FIG. 8
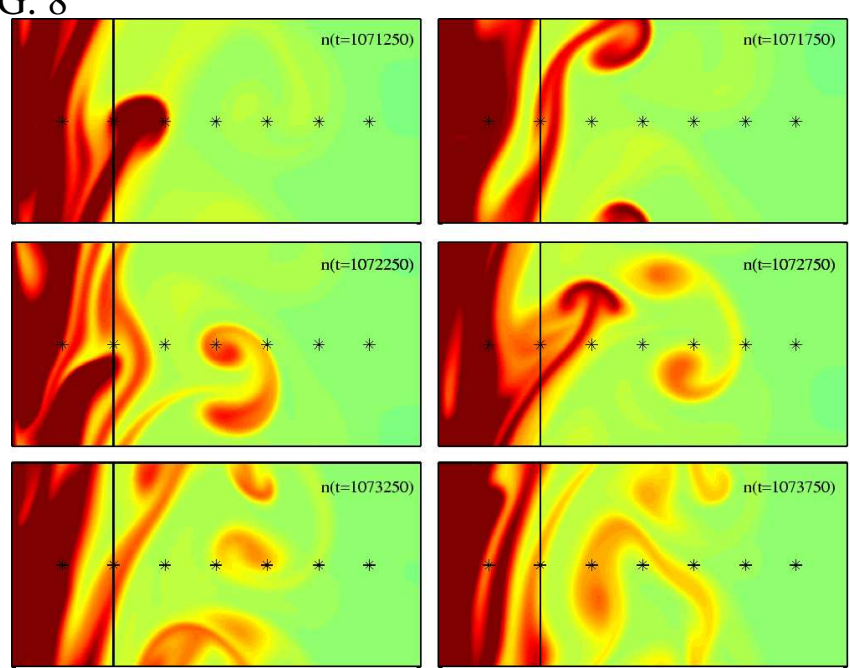

FIG. 9

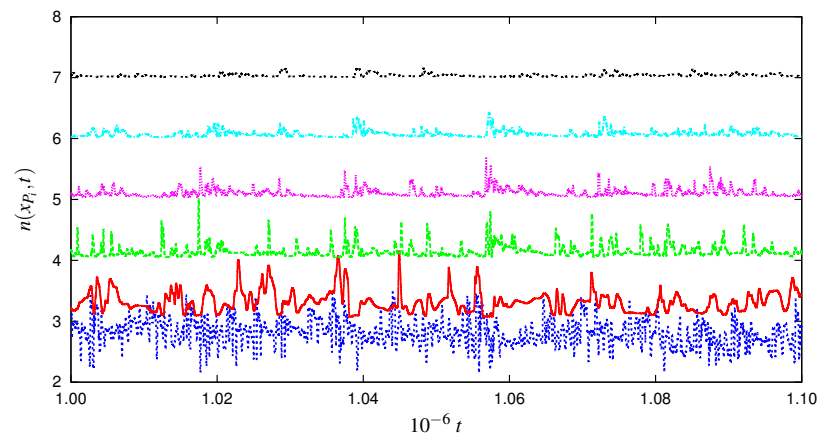

FIG. 10 


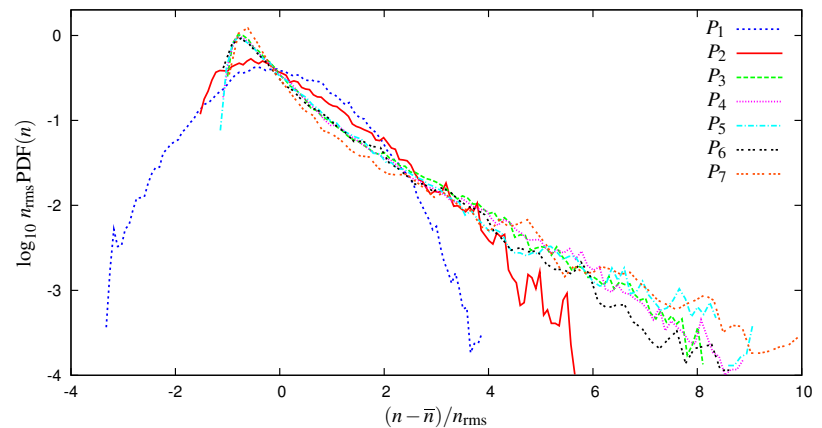

FIG. 11

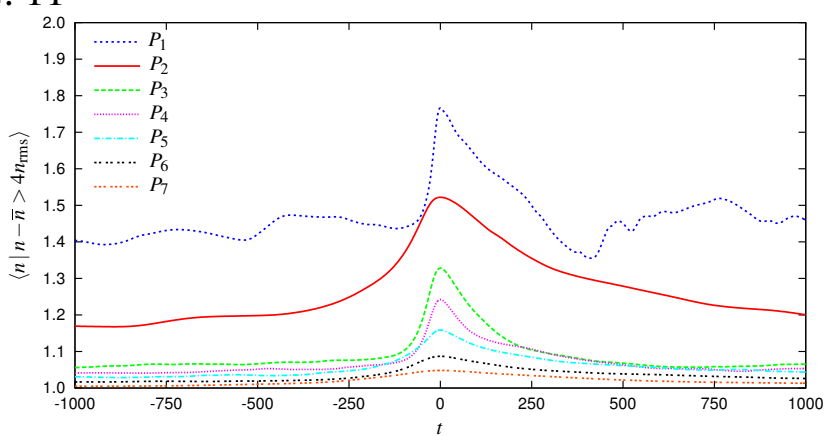

FIG. 12

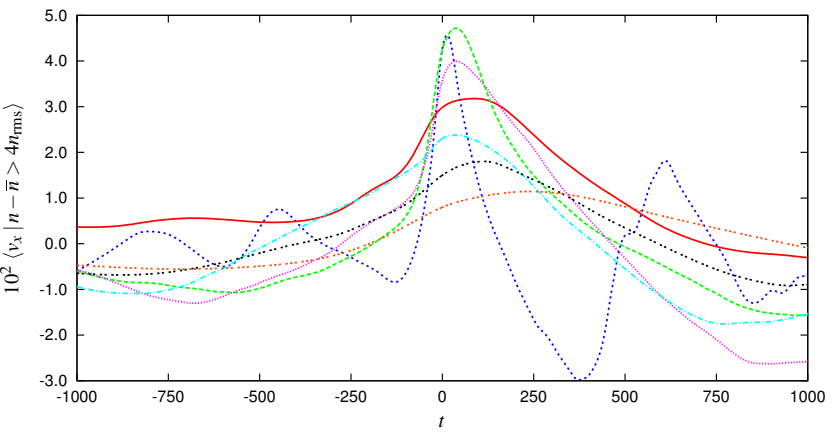

FIG. 13

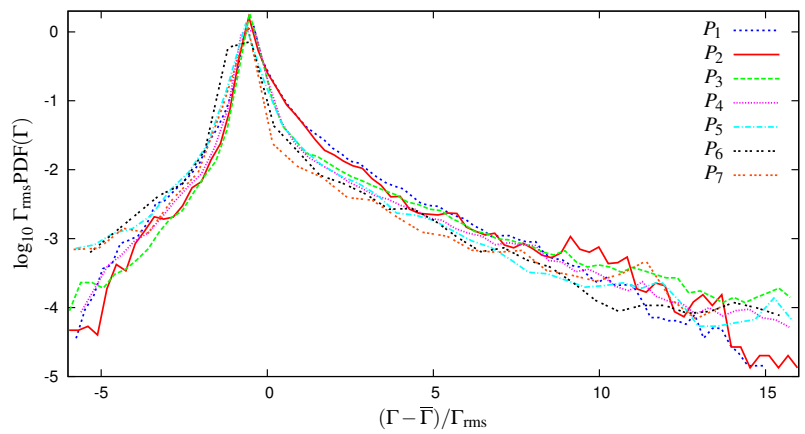

FIG. 14 


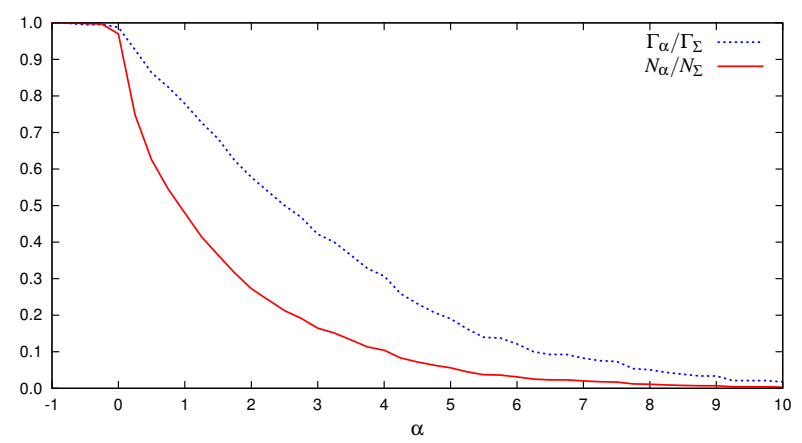

FIG. 15

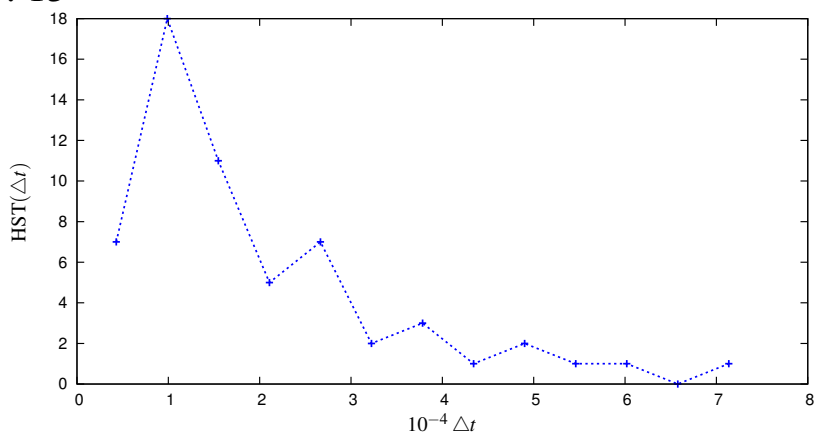

FIG. 16 